series, where 16-phenoxytetranor-PGE methyl-sulphonyleamide (Schering, Pfizer), 16, 16-dimethyl trans $\Delta^{2} \mathrm{PGE}_{1}$ methylester (ONO) and 9-deoxo-16, 16-dimethyl-9-methylene $\mathrm{PGE}_{2}$ (Upjohn) have been tested for use in both early (including menstrual regulation) and late abortion. The new compounds seem to produce fewer gastro-intestinal side effects and can be given intramuscularly, vaginally or orally. M. Bygdeman (Karolinska Institute, Stockholm) reported successful abortions in 10 of 12 women in the second trimester after oral doses of $25 \mathrm{mg}$ of 9-deoxy-16, 16-dimethyl-9-methylene $\mathrm{PGE}_{2}$ when given every third hour; the mean abortion time was $22 \mathrm{~h}$.

The conference illustrated the general applicability of prostaglandins and related compounds to all aspects of medicine. Just as prostacyclin and thromboxane synthetase inhibitors are now being developed as anti-coagulants one can look forward to major efforts to develop specific lipoxygenase inhibitors and leukotriene $\mathrm{C}$ antagonists for clinical use. The development of radioimmunoassays for leukotriene $\mathrm{C}$ should be of considerable diagnostic value in immunological diseases.

Marie Foegh is in the Department of Gynecology and Obstetrics, Herlev Hospital, University of Copenhagen.

\section{Sugars and intracellular recognition}

\section{from $M$. Geisow}

STUDIES of the biosynthesis and structure of membrane glycoproteins have reached maturity in the past few years. The longsuspected role of sugars in cell-surface recognition processes has been established with the identification of specific receptors. However, the reason for the glycosylation of many soluble secreted proteins (plasma proteins and hormones for example) is still not at all clear. Do such conjugated sugars have analogous extracellular recognition functions? In 1965, while considering the selective export of secreted proteins, Eylar ( $J$. theoret. Biol. 10, 89) hypothesised that conjugated sugars formed part of a primitive mechanism of export from cells. This early idea was right to the extent that it predicted the existence of an export 'signal', but wrong in the sense that this signal has turned out to be a peptide rather than an oligosaccharide (see News and Views 272, 308; 1978). In eukaryotic cells, newly synthesised proteins entering the lumen of the rough endoplasmic reticulum (RER) by virtue of this signal peptide must

$M$. Geisow is a member of the scientific staff at the National Institute for Medical Research, Mill Hill.

\title{
Yes, NGF is an enzyme
}

IN a recent article in the News and Views section of this Journal, P. Calissano and R. Levi-Montalcini have questioned the validity of our work on the proteolytic activity of nerve growth factor (NGF), and in particular, its ability to activate plasminogen ${ }^{1}$. Because their article contains misinformation, and because several of our papers on the points raised by Calissano and Levi-Montalcini were overlooked, we wish to respond as follows.

In 1977 we observed that crude homogenates of mouse submandibular glands contain multiple molecular-weight forms of $\mathrm{NGF}^{2}$. The largest of these, with mass 116,000 , was completely purified ${ }^{2}$ and later shown to be a specific proteolytic enzyme that can activate plasminogen ${ }^{3}$. It is also the predominant form of NGF which is secreted in high concentrations in mouse saliva ${ }^{4}$. Calissano and Levi-Montalcini state that this form of NGF "... exhibits a protease activity with an almost evanescent degree of specificity for plasminogen . . .". Yet, as shown in Figs 1,3 , and 4 in reference 3 there is nothing about the proteolytic activity of this species of NGF which indicates that it is transient. Rather, the enzyme activity is real, persistent, and highly specific. NGF is indeed much less active than urokinase in activating plasminogen, and we clearly pointed that out. We also stated that plasminogen might not be the natural (physiological) substrate for NGF - only that it was susceptible to activation by this protease $^{3}$.

With regard to the possibility that the protease associated with our NGF preparations might be a nonspecific contaminant ${ }^{1}$, Fig. 4 in reference 3 presents strong evidence that this is not the case. This figure shows that when our preparations of the 116,000 molecularweight form of NGF are examined by gel electrophoresis, all of the protein, all of the enzyme activity, and all of the immunoassayable NGF activity migrate with identical electrophoretic mobilities - as a single component.

More recent studies have revealed that this form of NGF exhibits some unusual features which are perhaps even more interesting than its plasminogenactivating property. For example, we have now shown that NGF, as isolated from mouse salivary glands, exists as a

nevertheless possess further chemical or structural attributes which will determine their ultimate fate. For example, complex enzymic processing follows the biosynthesis of hormone precursors like ACTH/endorphin (Mains et al. Proc. natn. Acad. Sci. U.S.A. 74, 3014; 1977). Furthermore, molecules within the ER/Golgi system, whether processed or zymogen and further, that this zymogen is converted to fully active enzyme by autocatalytic self-activation ${ }^{5}$. The zymogen itself is largely, if not entirely, enzymically inactive. Only after autocatalytic activation is full enzyme activity expressed ${ }^{5}$. This finding is probably the strongest evidence that the protease activity of NGF is specific and that it does not stem from a contaminant.

With regard to the comment of Calissano and Levi-Montalcini that the NGF activity might be "stuck" nonspecifically to other proteins present in the salivary gland, we must point out that if this is so, then it is "stuck" extremely tightly, it is "stuck" in constant ratio from preparation to preparation, and it is also "stuck" as it is naturally secreted in saliva ${ }^{4}$. All of this goes to the heart of an elementary problem of protein chemistry; namely, what constitutes the definition of a true molecular species. All of our results outlined above suggest that the NGFzymogen is such a species, and we know of no published evidence which indicates otherwise.

Finally, we should like to point out that of the several (at least six) forms of NGF present in salivary gland extracts, only the 116,000 molecular weight protein is stable $^{3}$. All of the other forms are degradation products of this single species $^{2}$. This includes the 2.5S-NGF (molecular weight 26,000 ) referred to by Calissano and Levi-Montalcini ${ }^{1}$. In fact, 2.5S-NGF accounts for less than $10 \%$ of the total NGF found in the salivary gland, and this minor component is the one which has been used in most experiments on NGF over the past twelve years.

The proteolytic activity of the 116,000 molecular weight form of NGF is a real property of this protein, and this fact, as well as the autocatalytic activation reaction, is going to have to be taken into account before the true physiological function of NGF can be understood.

MICHAEL YOUNG

Department of Biochemistry and Molecular Biology, University of Florida College of Medicine, Gainesville, Florida

1. Calissano, P \& Levi-Montalcini, R. Nature, News \&

2. Young, M., Saide, J.D. Murphy, R.A. \& Blanchard, M.H. Biochemistry, 17, 1490 (1978).

Orenstein, N.S. Dvorak, H.F. Blanchard, M.H. \& Young. Orenstein, N.S. Dvorak, H.F. Blanchard, M.H. \& Y
M. Proc. natn. Acad. Sci. U.S.A. 75, 6597 (1978). Murphy, R.A. Saide, J.D. Blanchard, M.H. \& Young, M Proc. natn. Acad. Sci. U.S.A. 74, 2672 (1977). 5. Young, M. Biochemistry 18, 3050 (1979).

not, need to be packaged in a different manner according to whether they are lysosomal, secreted or membrane proteins. Recent experimental work suggests the participation of conjugated carbohydrate in these intracellular recognition processes.

Melchers (Biochemistry 12, 1471; 1973) found that the glycosylation inhibitor 2-deoxy-D-glucose prevented migration of 